University of Nebraska - Lincoln

DigitalCommons@University of Nebraska - Lincoln

Mechanical \& Materials Engineering Faculty

Publications

Mechanical \& Materials Engineering

Department of

6-2005

\title{
Finite element analysis of covered microstents
}

\author{
Linxia Gu \\ University of Nebraska-Lincoln, gul@fit.edu \\ Swadeshmukul Santra \\ University of Florida \\ Robert A. Mericle \\ University of Florida \\ Ashok V. Kumar \\ University of Florida, akumar@ufl.edu
}

Follow this and additional works at: https://digitalcommons.unl.edu/mechengfacpub

Part of the Mechanical Engineering Commons

Gu, Linxia; Santra, Swadeshmukul; Mericle, Robert A.; and Kumar, Ashok V., "Finite element analysis of covered microstents" (2005). Mechanical \& Materials Engineering Faculty Publications. 39.

https://digitalcommons.unl.edu/mechengfacpub/39

This Article is brought to you for free and open access by the Mechanical \& Materials Engineering, Department of at DigitalCommons@University of Nebraska - Lincoln. It has been accepted for inclusion in Mechanical \& Materials Engineering Faculty Publications by an authorized administrator of DigitalCommons@University of Nebraska Lincoln. 
Published in Journal of Biomechanics 38:6 (June 2005), pp. 1221-1227; doi: 10.1016/j.jbiomech.2004.06.008

Copyright (C) 2004 Elsevier Ltd. Used by permission.

Accepted June 28, 2004; published online August 12, 2004.

\title{
Finite element analysis of covered microstents
}

\author{
Linxia Gu, ${ }^{1}$ Swadeshmukul Santra, ${ }^{2}$ Robert A. Mericle, ${ }^{2}$ and Ashok V. Kumar ${ }^{1}$ \\ ${ }^{1}$ Department of Mechanical \& Aerospace Engineering, University of Florida, P.O. Box 116300, Gainesville, FL 32611-6250, USA \\ ${ }^{2}$ Department of Neurological Surgery, University of Florida, P.O. Box 100265, Gainesville, FL 32610-0265, USA \\ Corresponding author - A. Kumar, tel 352 392-0816, fax 352 392-1071, e-mail akumar@ufl.edu
}

\begin{abstract}
Currently available neuroendovascular devices are inadequate for effective treatment of many wide-necked or fusiform intracranial aneurysms and intracranial carotid-cavernous fistulae (CCF). Placing a covered microstent across the intracranial aneurysm neck and CCF rent could restore normal vessel morphology by preventing blood flow into the aneurysm lumen or CCF rent. To fabricate covered microstents, our research group has developed highly flexible ultra thin $(\sim 150 \mu \mathrm{m})$ silicone coverings and elastomerically captured them onto commercially available metal stents without stitching. Preliminary in vivo studies were conducted by placing these covered microstents in the common carotid artery of rabbits. The feasibility of using covered stents was demonstrated. However, the cover affected the deployment pressure and the stents failed occasionally during deployment due to tearing of the cover. Appropriate modeling of covered stents will assist in designing suitable coverings, and help to reduce the failure rate of covered microstents. The purpose of this study is to use the finite element method to determine the mechanical properties of the covered microstent and investigate the effects of the covering on the mechanical behavior of the covered microstent. Variations in the mechanical properties of the covered microstent such as deployment pressure, elastic recoil and longitudinal shortening due to change in thickness and material properties of the cover have been investigated. This work is also important for custom design of covered microstents such as adding cutout holes to save adjacent perforating arteries.
\end{abstract}

Keywords: covered stent, finite element analysis, simulation, silicone, aneurysm, arteriovenous fistula

\section{Introduction}

Intracranial aneurysms and intracranial carotid-cavernous fistulae (CCF) are vascular malformations that occur in the intracranial compartment. An aneurysm is an abnormal dilation of a portion of an artery due to a weakening in the vessel wall either congenitally or by disease. The two main types of aneurysms that occur are saccular (berry) and fusiform aneurysms. Saccular aneurysms have a neck and involve part of the circumference of the wall whereas fusiform aneurysms do not have a neck and encompass the entire wall. Intracranial aneurysms can rupture and hemorrhage into the brain causing stroke with severe disability or death. An intracranial CCF is an abnormal direct connection between the intracranial carotid artery and the adjacent intracranial cavernous sinus (vein). In this situation, the high-pressure arterial blood is shunted directly into the low-pressure venous system without transversing the lengthy capillary bed. A CCF can cause venous hypertension, which can lead to a massive swollen and injected eye (proptosis and chemosis), glaucoma, blindness, deformity and reduced blood perfusion to the brain.

Treatment of intracranial aneurysms has traditionally been accomplished by open craniotomy and aneurysm clipping. These surgical procedures are invasive and involve a high risk (Giannotta and Litofsky, 1995; Johnston et al., 2001). Many non-invasive endovascular procedures have been developed to treat lesions such as intracranial aneurysms, intracranial CCF, 
and other pathological diseases that require redirection or restriction of blood flow in the brain. Endovascular balloons have been used for occlusion of CCF and parent arteries for treatment of aneurysms (Laitinen and Servo, 1978; Debrun, 1979). The use of thrombogenic coils for intracranial aneurysms was first performed in 1989, although coils were previously used for carotid artery occlusion (Braun et al., 1985). Detachable coils are currently used for some saccular aneurysms with narrow necks, as the coils are placed within the aneurysm lumen, while maintaining patency of the parent artery. Many wide-necked or fusiform aneurysms cannot be treated with coils because the coils cannot be contained in the aneurysmal sac (Ewald et al., 2000). Bare stents to assist in coiling some wide-necked aneurysms was first described in 1998 (Mericle et al., 1998). However, bare stents will never be useful for treatment of some complex wide-necked aneurysms, fusiform aneurysms, or $\mathrm{CCF}$, because the blood flow is free to pass between the stent struts and into the aneurysm lumen or fistula rent.

Covered microstents could be used to treat intracranial aneurysms and CCF's, because the covering would prevent blood flow into the lesion. A covered stent could be placed in the parent artery to bridge the abnormality to occlude blood flow into the aneurysm lumen or CCF rents. The use of covered stents in the proximal carotid and vertebral arteries has been reported (Geremia et al., 1997; Schellhammer et al., 1999; Fontaine et al., 2001; Najibi et al., 2002; Marty et al., 2002). For the intracranial application, the covered stent has to be miniaturized to fit the smaller, more complex arteries in the brain. The covered microstent should also be highly flexible and maneuverable for smooth navigation because of the increased tortuosity in the intracranial arteries. This requires that the covering material should be thin, flexible and should not move relative to the stent during or after placement. In our research group, a new kind of seamless covered microstent was developed. In order to most effectively design appropriate covered microstents, the mechanical properties of both stent and covering must be investigated. Clinical application of covered microstents would be dramatically improved if we understood and could predict how the mechanical properties of the covered stent would behave during deployment. The proper numerical analysis could help in designing covered stents and provide design-driven selection of suitable materials.

In this work, an ultra thin silicone covering of appropriate size was mounted onto a small Palmaz-Schatz stent before deployment. The effects of the covering on the primary mechanical performance of the stent (deployment pressure, elastic recoil and longitudinal shortening) was investigated by using finite element (FE) method. The results were compared to the mechanical properties of the microstent without covering. This is necessary to determine optimal deployment pressures, elastic recoil and longitudinal shortening for each desired diameter of artery where the covered stent would be placed in an intracranial artery.

FE analysis has been used to study mechanical properties of metallic stents, such as deployment pressure, elastic recoil and flexibility. Several studies have addressed the effects of stent geometries, the interaction between the stent and catheter balloon, or interactions between the stent and arterial wall. Auricchio et al. (2000) studied the biomechanical interaction between a balloon-expandable stent and a stenotic artery. Etave et al. (2001) determined the exact mechanical characteristics of two different types of stents: tubular stents and coil stents. Migliavacca et al. (2002) investigated the effects of different geometrical parameters of a typical diamond-shaped coronary stent on the mechanical performance, and gave some suggestions for optimizing stent shape and performance. Rogers et al. (1997) studied a 2D balloon-artery interaction. However, no numerical analysis of covered stents has been published. This work uses FE method to predict the mechanical properties of covered microstents, which is a critical step towards using these covered microstents to treat many difficult aneurysms and CCF.

\section{Preliminary animal studies}

The effect of covered stent placement was initially evaluated by histological analysis of the normal common carotid artery (CCA) in the New Zealand White (NZW) rabbit model. In a typical procedure, a vascular sheath was placed in the femoral artery of a NZW rabbit. Using endovascular techniques, a silicone-covered balloon-expandable stent device was navigated through the vasculature towards the CCA. The device was deployed within the vessel and angiography was performed to confirm patency. Following the device placement, the animal was monitored for six weeks. Angiography of the stented vessel was then performed to reveal angiographic patency. The animals were then euthanized and the vessels harvested for histological examination. Under this preliminary investigation, six animals were selected. In three cases, the covering ripped during stent deployment (at approximately 4 atm pressure) and stenosis occurred in one case. For the other cases, the placement of these covered microstents in the rabbit CCA resulted in minimal neointimal proliferation, which demonstrated the feasibility of using covered stents. Moreover, the covered stent device was easily navigated through the vasculature system, which demonstrates flexibility and maneuverability of this covered microstent.

In our animal study, we used approximately $150 \mu \mathrm{m}$ thick Silastic ${ }^{\circledR} \mathrm{T} 2$ silicone tubing elastomerically captured on balloon expandable metal stent. Although the 
stent is well optimized for clinical applications, there will be variations in the mechanical properties such as deployment pressure, elastic recoil etc. when it is covered with elastomeric covering. In general, irrespective of the type of metal stent, the alteration in mechanical properties of covered stent could be directly correlated to the mechanical properties of coverings. For intracranial applications, it is preferred to select ultra-thin covering in such a way that the covering will have minimal impact on the flexibility and maneuverability of the balloon-expandable stent system. However, to select appropriate thickness and material, it is not practical to perform repeated and expensive mechanical testing of covered stents with coverings of various elastomeric materials and thickness. Therefore, it is necessary to perform FE analysis to assist in designing stent coverings and selecting materials for the cover.

\section{Analysis and modeling}

An intracranial artery segment of $2.9 \mathrm{~mm}$ lumen diameter with a $4 \mathrm{~mm}$ long fusiform aneurysm is plotted (Figure 1). A covered microstent is placed as shown, across the aneurysm neck, and inflated by the balloon to $3 \mathrm{~mm}$ diameter to treat this lesion.

\subsection{Geometry and material properties}

A balloon-expandable Palmaz-Schatz microstent PS154 (Johnson \& Johnson, Warren, NJ, USA) was modeled in this study. It is a hollow tube with laser cut slots (Figure 2). The modeled stent has an outer diameter of $1.47 \mathrm{~mm}$, a length of $8.06 \mathrm{~mm}$ and a thickness of $0.1 \mathrm{~mm}$. There are 2 slots in the longitudinal direction and 12 slots circumferentially. The dimension of each slot is $3.62 \mathrm{~mm} \times 0.22 \mathrm{~mm}$. The distal strut length is $0.3 \mathrm{~mm}$, the inner strut length is $0.22 \mathrm{~mm}$, and the metal strut width is $0.14 \mathrm{~mm}$.

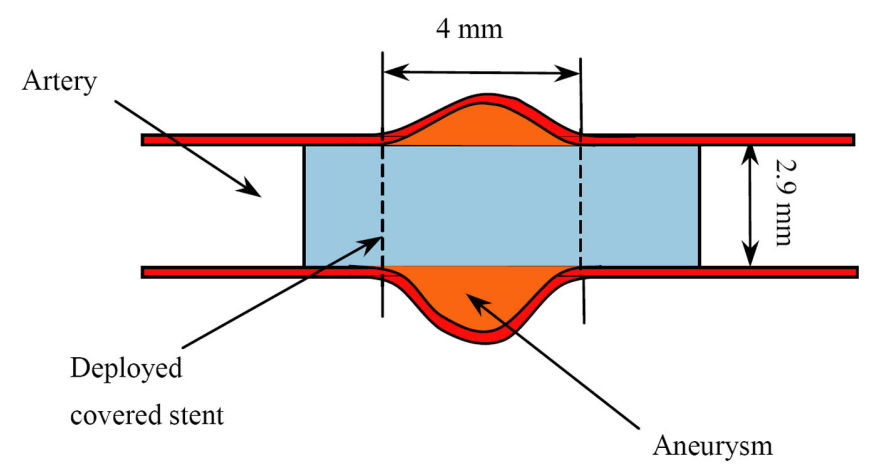

Figure 1. Schematic diagram of an intracranial artery segment with a fusiform aneurysm.

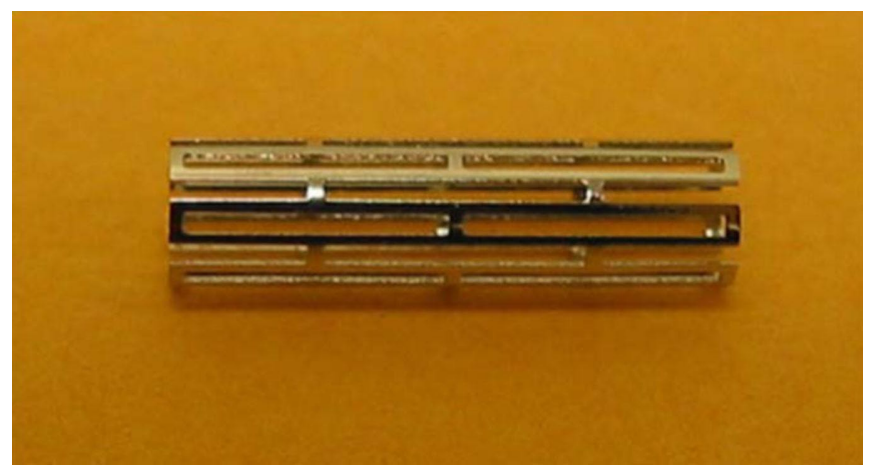

Figure 2. Photographs of Palmaz-Schatz balloon expandable microstent in its constricted form.

Plastic properties of the material such as yield stress and hardening depend upon previous work hardening or history of deformation. In the absence of detailed material information from the manufacturer, the material properties of the stent were adopted from published literature (Auricchio et al., 2000; Migliavacca et al., 2002). The microstent was assumed to be made of $316 \mathrm{LN}$ stainless steel. The material properties that were used for the analysis are: Young's modulus $E=196 \mathrm{GPa}$; Poisson ratio $v=0.3$; Yield stress $\sigma_{\mathrm{Y}}=205 \mathrm{MPa}$; ultimate stress $\sigma_{\mathrm{M}}$ $=515 \mathrm{MPa}$ and the corresponding ultimate strain $\varepsilon_{\mathrm{M}}=$ $60 \%$. The plastic behavior of the microstent was modeled assuming linear isotropic hardening between yield stress and ultimate stress.

Our model considered an ultra thin elastomeric tubular covering captured on the metal PS154 stent. In order to address the frequent covering migrations described in the literature (Schellhammer et al., 1999), we left $0.1 \mathrm{~mm}$ of the stent uncovered at each end of the $8.06 \mathrm{~mm}$ long microstent. After deployment, the uncovered stent ends will have a subtle trumpet-like flare. The uncovered portion of the stent provides a better grip on the arterial wall to prevent covered microstent migration.

Silicone covering were made from Silastic ${ }^{\circledR} \mathrm{T} 2$ base and Silastic T2 curing agent (Dow Corning ${ }^{\circledR}$, Midland, MI). The material properties such as elastic modulus and rupture stress/strain of the silicone covering were measured using an Instron model 4301 testing instrument. Complete stress versus strain profiles for the ten samples was thus obtained (Figure 3 ). The average modulus was $2.47( \pm 0.14) \mathrm{MPa}$, and the strain at rupture was $259( \pm 19.12) \%$. In our FE modeling, we assumed that the cover has linear elastic behavior with Young's modulus of $2.47 \mathrm{MPa}$ and Poisson's ratio of 0.3 .

\subsection{Simulation}

FE analysis is widely used as a tool to provide cost-effective information on product design and test as part of 


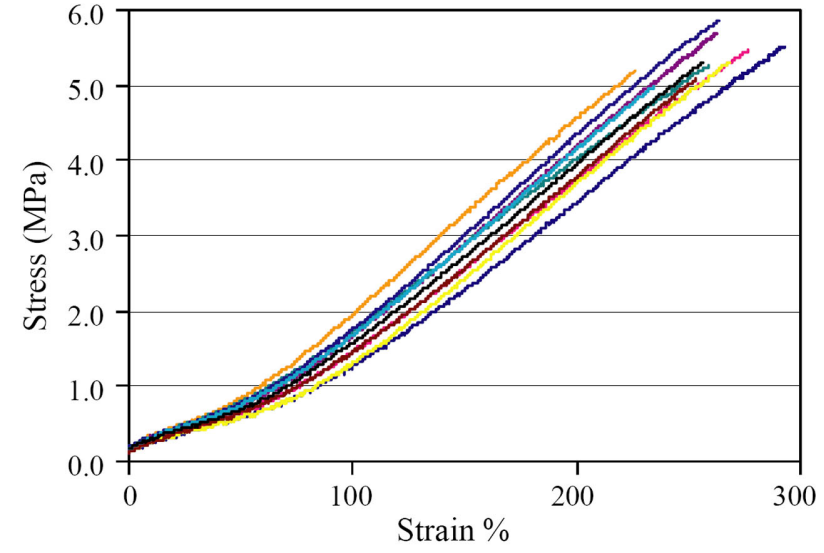

Figure 3. Nominal stress versus strain curve for Silastic ${ }^{\circledR} \mathrm{T} 2$ silicone (10 samples).

the product development process. Considering the nonlinear large plastic deformation of the metallic stent and the tight contact between the stent and the covering, we used commercial FE software: ABAQUS 6.3 (2002), from Hibbitt, Karlsson \& Sorensen, Inc., Rhode Island, USA.

The 3D geometry of the microstent and covering were developed using commercial software I-DEAS 9 (EDS, Texas, USA) that has the capability to create complex solid and surface models, as well as mesh. The whole stent was meshed with 4-node general shell elements S4R (Figure 4a). Due to the circular symmetry of the geometry it is possible to model just $1 / 12$ th of the stent instead of modeling the entire stent. Even though identical results were obtained by modeling $1 / 12$ th of the stent, we have shown only results and figures obtained using models of the complete stent since they are easier to understand and interpret for non-engineers. Note that the stent thickness to radius ratio is approximately $1 / 7$, therefore we have used a thick shell element that accounts for transverse shear stresses and strains. If 8-node hexahedral (3D) elements were used, it would be necessary to use a large number of elements through the thickness of the stent to accurately capture the stress variation through the thickness. The complete geometry of the stent was discretized into 2730 elements with average length of $0.0796 \mathrm{~mm}$ and 3936 nodes. The covering was discretized into 6272 elements and 6336 nodes with the element length of $0.08 \mathrm{~mm}$.

Careful observation of the in vivo stenting experiments in our lab showed that the balloon is almost uniformly inflated except at two ends, and the stent is expanded by the uniformly inflated part of the balloon. The free ends of the stent are easier to expand than the central part. Thus, the ends of the stent expand faster at the beginning of the expansion. The central portion of the stent continues to be expanded by the balloon until the stent is almost evenly expanded (Figure $4 b$ ). The balloon transmits the internal pressure uniformly to the stent during the expansion. Therefore, a uniform

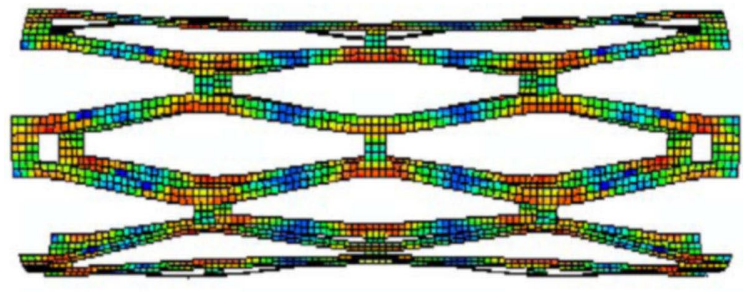

(a)

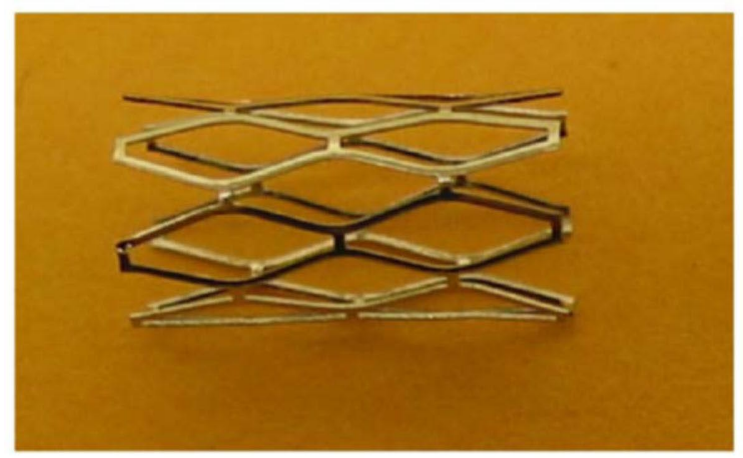

(b)

Figure 4. Free expanded microstent at 3-mm-diameter with a diamond shape slot (a) FE element results, (b) in vitro experiment photo.

pressure was applied in our model on the internal surface of the covered microstent. During loading, pressure was increased until the final diameter of the stent reached the value of $3 \mathrm{~mm}$ and then the pressure was unloaded to study recoil. All rotational degrees of freedom were fixed for the nodes at both ends of the stent. A non-sliding contact between the covering and the microstent was prescribed. The initial stress in the stent and covering were neglected. The different thickness of 0.08 , $0.1,0.12$ and $0.15 \mathrm{~mm}$ for the covering were tested under radial pressure load.

\section{Results}

The primary objective of this work is to compare the mechanical properties of the covered microstent with stents that do not have coverings (or bare stent). Stents were expanded by uniform internal pressure that was applied in small increments. Large structural deformation and material non-linearities can pose difficulties due to non-convergence after certain amount of deformation. ABAQUS provides automatic mechanisms for stabilizing this kind of problems through the automatic addition of volume-proportional damping to the model. Even so the maximum deformation that can be simulated using FE analysis is restricted due to non-convergence if the mesh is severely distorted.

A simple in vitro experiment was performed to study the expansion of the stent. A bare balloon-expandable 
Palmaz-Schatz stent was expanded by increasing the pressure in very small steps and the diameter of the stent was recorded at each pressure. Figure 4(b) shows the expanded stent at the end of the experiment. During the expansion the slots in the stent expand to a diamond shape allowing the stent to expand significantly. After the stent starts deforming plastically the rate of pressure increase needed to continue expanding the stent was very low indicating that the stent material does not work harden significantly.

A FE model of the stent was created and expanded using uniform pressure. The expanded stent has a diamond-shaped slot after expanding from the initial outer diameter of $1.47-3 \mathrm{~mm}$. In he final expanded state, the diameter at the ends of the stent is slightly larger than at the center. Figure 4(a) shows the expanded stent obtained by the finite element analysis. The shape of the expanded stent predicted by the analysis matches the experimentally observed shape (Figure 4). Furthermore, from FE analysis results, we can see that there are stress concentrations at the joints, as expected.

The deformation of the covered stent was also obtained under the uniform internal pressure (Figure 5). It is clear that the covering expands together with the stent in the areas where they are in contact, indicating that the contact model worked as expected. The uncovered regions at the ends of the stent expanded more than the covered part. This prevents any movement of the covering with respect to the stent after deployment. This agrees with the observations during lab tests described in Section 2.

The diameter of the bare stent (at the mid point) computed by the FE analysis was plotted as a function of the applied pressure (Figure 6). This plot shows that the pressure jumped dramatically from 0 to $2.6 \mathrm{~atm}$ mostly during the elastic deformation when the diameter increased by $0.1 \mathrm{~mm}$ for the bare stent. The ratio of pressure change to diameter change is $26.2 \mathrm{~atm} / \mathrm{mm}$. Then the curve becomes almost flat during the plastic deformation. The stent experiences large plastic de-

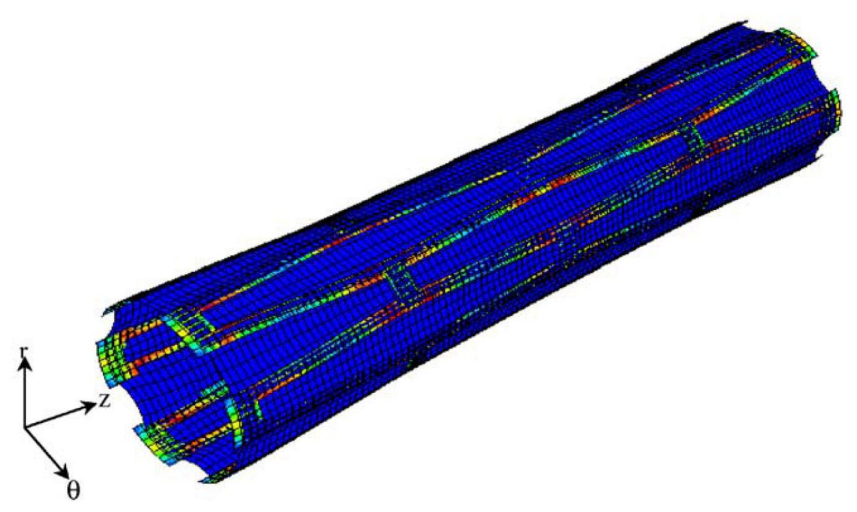

Figure 5. Deformation of covered microstent. formation from 1.57 to $3 \mathrm{~mm}$ in diameter. The pressure increased by a relatively small amount (just $1.18 \mathrm{~atm}$ ) during this expansion of the stent and the ratio of pressure increase to diameter increase is only $0.82 \mathrm{~atm} /$ $\mathrm{mm}$. The pressure does not increase much during this plastic deformation because of the low rate of hardening. This matches with experimental observations that the pressure does not increase much after the stent starts deforming plastically. After being unloaded, the stent recoils back along a line parallel to the elastic portion of the loading curve.

For the covered microstent, the deployment pressure versus deformation plot (Figure 7a) has the same trend as the plot for the bare stent. The pressure jumps during the first $0.1 \mathrm{~mm}$ deformation. Beyond $0.1 \mathrm{~mm}$ deformation $(D=1.57 \mathrm{~mm})$, the pressure does not increase significantly. For comparison, the figure includes the pressure versus deformation plot for the bare stent and a $0.1 \mathrm{~mm}$ covering when it is expanded without mounting on a stent. One of the curves corresponds to a covering of 0.1-mm-thickness with a lower Young's Modulus, $E$ _cover $=1.8 \mathrm{MPa}$. This curve illustrates the effect of changing the material of the cover to a softer material. It is clear that a thinner and softer covering is preferred in the design of the covered microstent because the deployment pressure decreases.

When a 0.1-mm-thick covering was used, the required pressure increased by approximately 30\% compared to the bare stent at a deformed diameter of $1.57 \mathrm{~mm}$. Note that the required pressure to expand covered stent is not equal to the sum of the pressure for bare stent and the covering. This is due to the non-sliding contact between the microstent and the covering. Different covering thicknesses $(0.08,0.1,0.12$ and $0.15 \mathrm{~mm})$ were tested. The deployment pressure required versus the thickness of the covering was plotted using the FE simulation results (Figure $7 \mathrm{~b}$ ). The two curves correspond to the deployment pressure required to expand the covered stent to a final diameter of 1.54 and $1.57 \mathrm{~mm}$, respectively. The results in the figure predict

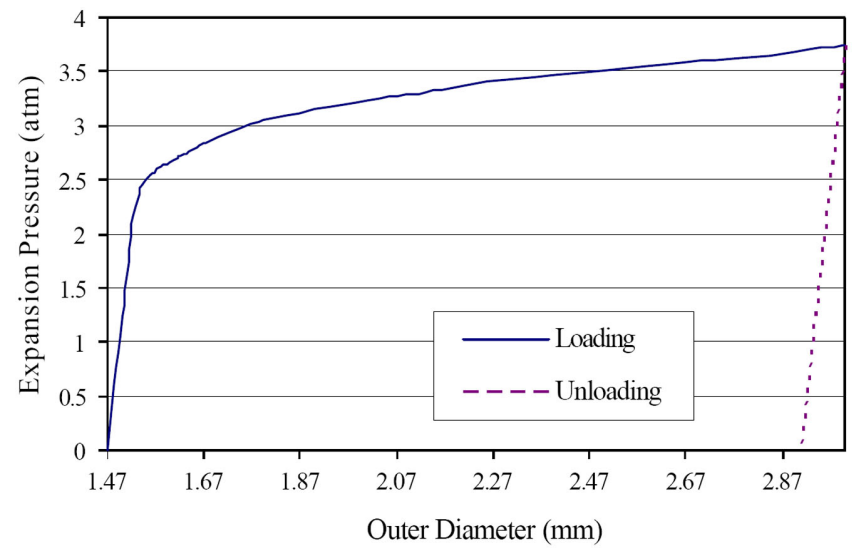

Figure 6. Deployment pressure as a function of the central outer diameter of bare stent. 


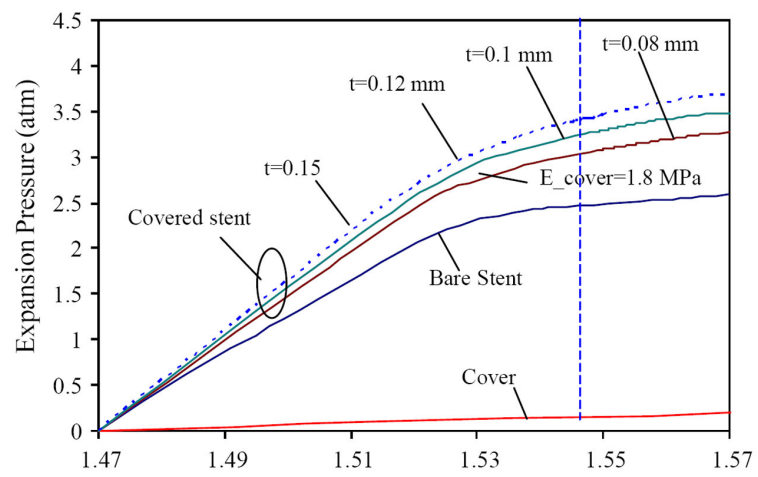

(a)

Outer Diameter (mm)

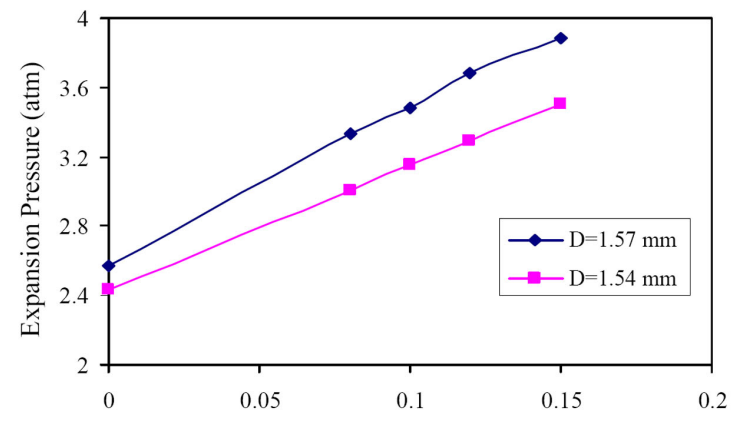

(b)

Cover thickness (mm)

Figure 7. (a) Deployment pressure required to reach 1.57-mmdiameter in the central area of the stent $\left(P_{1.57 \mathrm{~mm}}\right)$ for cases with different covering thickness $t$, different Young's modulus of the covering E_cover; (b) $P_{1.57 \mathrm{~mm}}$ and $P_{1.54 \mathrm{~mm}}$ versus covering thickness.

that the required deployment pressure increases almost linearly with the thickness of the covering.

Other factors to be considered in the design of covered microstents include longitudinal shortening and elastic recoil. Longitudinal shortening is the relative difference between the initial length of the stent $L_{0}$ and the expanded length $L_{\text {load }}$. Elastic recoil is defined as the difference between the diameter at expanded state $D_{\text {load }}$ and stent diameter after withdrawing the deployment pressure $D_{\text {unload }}$. The longitudinal shortening remained almost unchanged with different covering thicknesses (Figure 8a). But there is a large difference between the covered microstents and bare stent. This is because the covering alone has a much larger longitudinal shortening than the bare stent due to the large difference in elastic modulus. Using Hooke's law the strain in the longitudinal direction can be expressed as $\varepsilon_{z z}=(1 / E)\left(\sigma_{z z}\right.$ $\left.v \sigma_{x x}-v \sigma_{y y}\right)$, where $z$ direction is assumed to the longitudinal direction and $\sigma_{x x^{\prime}} \sigma_{y y^{\prime}} \sigma_{z z}$ are the stress components in the $x, y$, and $z$ directions, respectively. Clearly, the strain would be much larger for a material with smaller Young's modulus, E even when the Poisson's ratio, v, is the same. Therefore, when the covering is captured onto the stent, it causes a larger longitudinal shortening for the covered stent. The large difference in longitudinal shortening between the cover and stent may

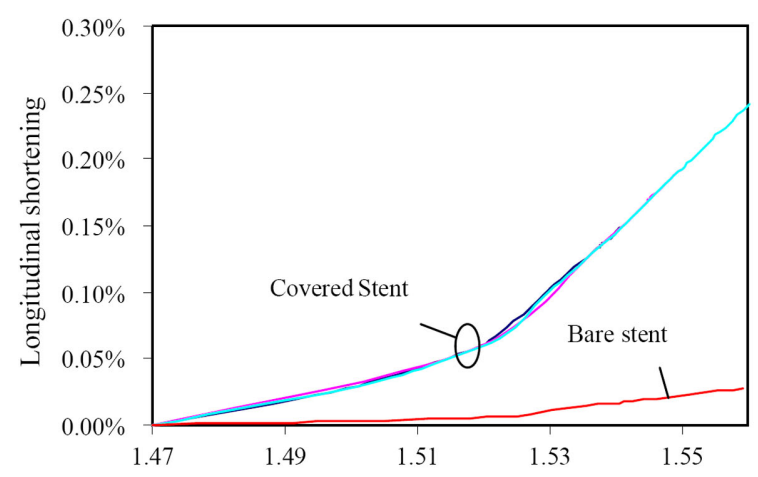

(a)

Outer Diameter (mm)

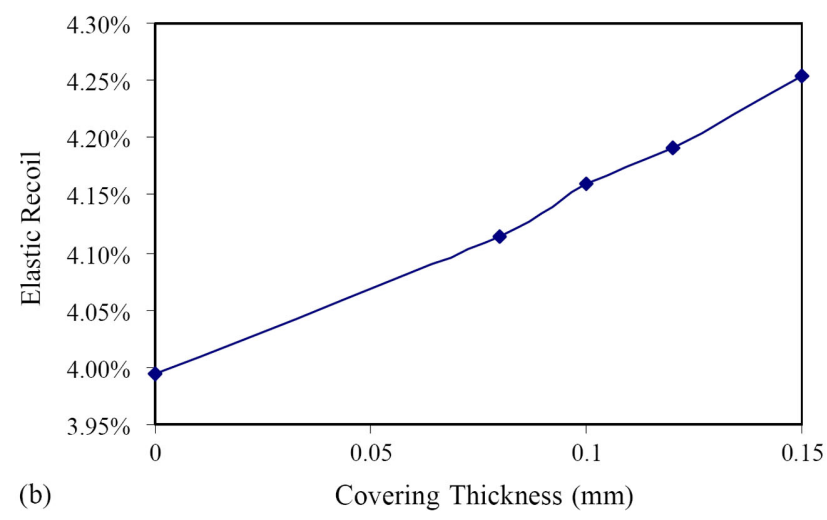

Figure 8. (a) Longitudinal shortening at different deformation diameter for different covering thickness, (b) elastic recoil for different covering thickness.

cause slippage between the two for some covering materials if the sliding friction is low. In our experiments, the covered stents were made by elastomerically capturing a thin Silicone cover (a tubular polymer sheath) onto the metal stent. In the in vivo experiments, no slippage of the cover was observed during the expansion of the stent. Therefore, it was assumed in the FE model that there is no relative slip between the cover and the stent. The elastic recoil determines the final diameter of the covered stent after the balloon withdraws. We can see that the elastic recoil increases almost linearly with covering thickness (Figure 8b).

\section{Discussions}

The results above show that the contact between the stent and the covering influences the mechanical behavior of the covered stent. The deployment pressure required to inflate the covered stent was found to be proportional to the thickness of the covering. Covered stent was found to have larger longitudinal shortening than a bare stent but it does not vary significantly with change in cover thickness. A thinner covering with low Young's modulus is desirable because it has lower deployment pressure and elastic recoil. However, a thinner covering 
is more likely to rupture during expansion. Therefore, it is important to ensure that the covered microstent can be expanded to the desired diameter without rupturing the covering. This trade off needs to be further investigated to improve the design of the covered microstent.

This work is important for custom design of covered microstents such as adding cutout holes to save perforating arteries. Further modeling will be performed on the design of covering patches, other types of covered microstent, and different covering materials.

\section{Acknowledgments}

This work was supported by the University of Florida Opportunity Fund 2002, Grant No. 2050260.

\section{References}

ABAQUS 6.3, 2002 • ABAQUS 6.3-1 documentations, 2002. Hibbitt, Karlsson \& Sorensen, Inc., Rhode Island, USA.

Auricchio et al., $2000 \bullet$ F. Auricchio, M. D. Loretob, and E. Saccoc, Finite-element analysis of a stenotic artery revascularization through a stent insertion, Computer Methods in Biomechanics and Biomedical Engineering 00 (2000), pp. 1-15.

Braun et al., 1985 • I. S. Braun, J. C. Hoffman, W. J. Casarella, and P. C. Davis, Use of coils for transcatheter carotid occlusion, American Journal of Neuroradiol 6 (1985), pp. 953-956.

Debrun, 1979 • G. Debrun, Cerebral aneurysms: advances in diagnosis and therapy. In: H.W. Pia, C. Langmaid and J. Zierski, Editors, Special Therapeutic Problems, Springer, New York (1979), pp. 228-236.

Etave et al., 2001 - F. Etave, G. Finet, M. Boivin, J. Boyer, G. Rioufol, and G. Thollet, Mechanical properties of coronary stents determined by using finite element analysis, Journal of Biomechanics 34 (2001), pp. 1065-1075.

Ewald et al., 2000 - C. Ewald, D. Kühne, and W. E. Hassler, Bypass-surgery and coil-embolisation in the treatment of cerebral giant aneurysms, Acta Neurochirrurgica 142 (2000), pp. 731-738.

Fontaine et al., 2001 • A. B. Fontaine, J. J. Borsa, E. Hoffer, R. Bloch, and C. So, Evaluation of silicone as an endovascular stent membrane: in vivo canine studies, Cardiovascular and Interventional radiology 24 (5) (2001), pp. 324-328.
Geremia et al., 1997 • G. Geremia, M. Bakon, L. Brennecke, M. Haklin, and B. Silver, Experimental arteriovenous fistulas: treatment with silicone-covered metallic stents, American Journal of Neuroradiology 18 (2) (1997), pp. 271-277.

Giannotta and Litofsky, 1995 • S. L. Giannotta and N. S. Litofsky, Reoperative management of intracranial aneurysms, Journal of Neurosurg 83 (1995), pp. 387-393.

Johnston et al., 2001 S. C. Johnston, S. Zhao, R. A. Dudley, M. F. Berman, and D. R. Gress, Treatment of unruptured cerebral aneurysms in California, Stroke 32 (2001), pp. 597-605.

Laitinen and Servo, $1978 \bullet$ L. Laitinen and A. Servo, Embolization of cerebral vessels with inflatable and detachable balloons - technical note, Journal of Neurosurgery 48 (1978), pp. 307-308.

Marty et al., 2002 • B. Marty, A. J. Leu, A. Mucciolo, and L. K. von Segesser, Biologic fixation of polyester- versus polyurethane-covered stents in a porcine model, Journal of Vascular and Interventional Radiology 13 (6) (2002), pp. 601-607.

Mericle et al., $1998 \bullet$ R. A. Mericle, G. Lanzino, A. K. Wakhloo, L. R. Guterman, and L. N. Hopkins, Stenting and secondary coiling of intracranial internal carotid artery aneurysm, Neurosurgery 43 (1998), pp. 1229-1234.

Migliavacca et al., 2002 • F. Migliavacca, L. Petrini, M. Colombo, F. Auricchio, and R. Pietrabissa, Mechanical behavior of coronary stents investigated through the finite element method, Journal of Biomechanics 35 (2002), pp. 803-811.

Najibi et al., 2002 • S. Najibi, R. L. Bush, T. T. Terramani, E. L. Chaikof, A. B. Gunnoud, A. B. Lumsden, and V. J. Weiss, Covered stent exclusion of dialysis access pseudoaneurysms, Journal of Surgical Research 106 (1) (2002), pp. 15-19.

Rogers et al., 1997 • C. Rogers, D. Y. Tseng, J. C. Squire, and E. R. Edelman, Balloon-artery interactions during stent placement: A finite element analysis approach to pressure, compliance, and stent circulation. In Proceedings of the $23 \mathrm{rd}$ Annual Meeting of the Society for Biomaterials. New Orleans, La, 1997.

Schellhammer et al., 1999 • F. Schellhammer, A. K. Wahkloo, H. Nagursky, and M. Schumacher, Vein graft-coated stents for endovascular occlusion of canine experimental arteriovenous fistulae, Investigative Radiology 34 (1) (1999), pp. 22-27. 